\begin{tabular}{|c|c|}
\hline hommes $_{\& \text { migrations }}$ & $\begin{array}{l}\text { Hommes \& migrations } \\
\text { Revue française de référence sur les dynamiques } \\
\text { migratoires } \\
\mathbf{1 2 8 1} \text { | } \mathbf{2 0 0 9} \\
\text { France-Brésil sous l'angle des migrations et de } \\
\text { l'altérité }\end{array}$ \\
\hline \multicolumn{2}{|c|}{$\begin{array}{l}\text { Migrations internationales et populations } \\
\text { étrangères en France et au Brésil }\end{array}$} \\
\hline \multicolumn{2}{|c|}{$\begin{array}{l}\text { Édition électronique } \\
\text { URL: http://journals.openedition.org/hommesmigrations/372 } \\
\text { DOI : } 10.4000 / \text { hommesmigrations.372 } \\
\text { ISSN : 2262-3353 }\end{array}$} \\
\hline \multicolumn{2}{|c|}{$\begin{array}{l}\text { Éditeur } \\
\text { Musée national de l'histoire de l'immigration }\end{array}$} \\
\hline \multicolumn{2}{|c|}{$\begin{array}{l}\text { Édition imprimée } \\
\text { Date de publication : } 1 \text { septembre } 2009 \\
\text { Pagination : } 40-53 \\
\text { ISSN : } 1142-852 X\end{array}$} \\
\hline $\begin{array}{l}\text { Référence électroniqu } \\
\text { Hervé Théry, « Migratic } \\
\text { \& migrations [En ligne], } \\
\text { http://journals.opened }\end{array}$ & $\begin{array}{l}\text { ss internationales et populations étrangères en France et au Brésil », Hommes } \\
281 \text { | 2009, mis en ligne le } 29 \text { mai } 2013 \text {, consulté le } 14 \text { novembre } 2019 \text {. URL: } \\
\text { on.org/hommesmigrations/372; DOI : 10.4000/hommesmigrations.372 }\end{array}$ \\
\hline
\end{tabular}

Tous droits réservés 


\section{Migrations internationales et populations étrangères en France et au Brésil}

Par Hervé Théry, géographe, directeur de recherche au CNRS-Credal, professeur invité à l'université de São Paulo - USP

Une analyse des populations étrangères en France et au Brésil fait ressortir de profondes différences entre les deux pays, notamment en ce qui concerne les nationalités représentées et les mécanismes migratoires.

Alors que dans l'Hexagone les ressortissants des pays voisins d'Europe du Sud et des anciennes colonies sont toujours les plus nombreux, au Brésil

les origines migratoires connaissent depuis quelques décennies un net

changement. Si les pays qui avaient le plus alimenté l'immigration auX XIX' et XX' siècles l'emportent en nombre de ressortissants, le Brésil est désormais au centre de nouvelles migrations régionales. 
Une analyse cartographique fondée sur les données de l'INSEE ${ }^{(1)}$ et de l'IBGE ${ }^{(2)}$, son homologue brésilien, permet de mettre en lumière les situations contrastées de la France et du Brésil vis-à-vis des migrations internationales. Ces données s'appuient sur les recensements démographiques des deux pays. Il faut commencer par mettre en parallèle la répartition géographique et la composition des populations migrantes par nationalité. Dans le cas brésilien, la répartition des immigrations issues des flux du siècle dernier fait progressivement place à de nouveaux courants, provenant principalement du continent sud-américain, mais aussi de pays plus lointains, dont les habitants avaient jusque-là peu migré vers le Brésil.

L'analyse des autorisations de migration entre 2004 et 2008 montre des apports nouveaux, notamment par des arrivées en provenance des États-Unis et de divers pays asiatiques. À cette réorientation en fonction de l'origine des migrants correspond un changement de destination à l'intérieur du pays. Les migrants privilégient nettement les grandes métropoles du Sudeste, notamment São Paulo et Rio de Janeiro.

\section{Les étrangers en France et au Brésil}

Les étrangers recensés en France étaient en 2000, selon l'INSEE, 5618479 sur 58520688 habitants. Au Brésil, ils étaient, selon l'IBGE, 510067 non naturalisés et 173763 naturalisés sur 173 millions (contre 606624 non naturalisés et 161151 naturalisés en 1991 sur 161 millions). En 2009, on estime qu'ils sont environ 880 000. Le chiffre est en légère augmentation donc, mais, en proportion, bien inférieur à celui de la France, ce qui est a priori surprenant pour un pays qui a si lontemps été un pays d'immigration massive.

Dans les deux pays (cf. figures 1 et 2, p. 42), les populations étrangères se concentrent dans les grandes métropoles, mais de façon plus accentuée au Brésil, notamment à São Paulo et Rio de Janeiro. Une représentation cartographique non conventionnelle (en anamorphose, cf. figures 3 et 4, p. 43) montre plus clairement les concentrations de populations étrangères dans les régions centrales des deux pays.

Les six nationalités les plus représentées parmi les étrangers résidant en France sont issues des pays voisins de l'Europe du Sud (Portugal, Espagne et Italie) ou des anciennes colonies d'Afrique du Nord (Algérie, Maroc et Tunisie).

Au Brésil, on constate une forte représentation des mêmes pays d'Europe du Sud, d'où sont venus les principaux courants migratoires, notamment du Portugal, l'ancienne métropole. Deux autres pays ont alimenté les courants migratoires importants des $\mathrm{XIX}^{\mathrm{e}}$ et $\mathrm{XX}^{\mathrm{e}}$ siècles (l'Allemagne et le Japon), alors que le troisième est un pays voisin (l'Argentine). On est donc bien en présence des résultats des migrations passées, du temps où le Brésil voyait sa population croitre principalement grâce à l'immigration. 
42 Dossier | France - Brésil, sous l'angle des migrations et de l'altérité |

\section{Figure 1. Les étrangers en France}

Source INSEE - ๑ Hervé Théry, 2009.

Figure 2. Les étrangers au Brésil 
Figure 3. Les étrangers en France, anamorphose 
44 Dossier | France - Brésil, sous l'angle des migrations et de l'altérité |

Figure 5. Les six nationalités les plus représentées en France

Figure 6. Les six nationalités les plus représentées au Brésil 


\section{Le Brésil, pays d'immigration}

Contrairement à d'autres pays d'Amérique latine, comme le Pérou, le (futur) Brésil ne comptait pas de populations indiennes nombreuses à l'arrivée des Européens. L'essentiel de sa population lui est venu par immigration, à l'époque coloniale (1500 à 1822) ou postérieurement.

À l'époque coloniale, les principales migrations provenaient de la péninsule Ibérique, le pays étant fermé au commerce et aux migrations par la politique coloniale de la métropole, Lisbonne ou - durant la période où les deux pays étaient unis - Madrid. Après l'indépendance, sous l'Empire (de 1822 à 1889), une politique d'immigration depuis l'Europe avait été amorcée, en prévision de l'abolition de l'esclavage qui fournissait jusque-là l'essentiel de la main-d'ceuvre dans les plantations et les mines. De fait, une fois l'esclavage aboli, ce qui entraîna la chute de l'Empire, c'est l'immigration européenne qui assurait la croissance de la population et la prospérité du pays, alors en plein boom du café.

Ce flux migratoire resta très fort jusqu'aux années trente, puis chuta lors de la Seconde Guerre mondiale et ne reprit que brièvement et modestement après celleci. Les immigrés les plus nombreux, outre les Portugais et les Espagnols, qui continuaient à prendre la route de l'Amérique du Sud, furent les Italiens et les Allemands. Leurs pays étaient alors en plein processus d'unification et de transformation économique profonde, ce qui "libérait" des populations paysannes ne trouvant plus leur place dans les sociétés en cours de modernisation.

\section{Tableau 1. Migrants par nationalité}

\begin{tabular}{|c|c|c|c|c|c|c|c|c|c|}
\hline $\begin{array}{l}\text { Période } \\
\text { Nationalité }\end{array}$ & $\begin{array}{l}1884 \\
1893\end{array}$ & $\begin{array}{l}1894 \\
1903\end{array}$ & $\begin{array}{l}1904 \\
1913\end{array}$ & $\begin{array}{l}1914 \\
1923\end{array}$ & $\begin{array}{l}1924 \\
1933\end{array}$ & $\begin{array}{l}1945 \\
1949\end{array}$ & $\begin{array}{l}1950 \\
1954\end{array}$ & $\begin{array}{l}1955 \\
1959\end{array}$ & Total \\
\hline Italiens & 510533 & 537784 & 196521 & 86320 & 70177 & 15312 & 59785 & 31263 & 1507695 \\
\hline Portugais & 170621 & 155542 & 384672 & 201252 & 233650 & 26268 & 123082 & 96811 & 1391898 \\
\hline Espagnols & 113116 & 102142 & 224672 & 94779 & 52405 & 4092 & 53357 & 38819 & 683382 \\
\hline Autres & 66524 & 42820 & 109222 & 51493 & 164586 & 29552 & 84851 & 47599 & 596647 \\
\hline Japonais & - & - & 11868 & 20398 & 110191 & 12 & 5447 & 28819 & 176735 \\
\hline Allemands & 22778 & 6698 & 33859 & 29339 & 61723 & 5188 & 12204 & 4633 & 176422 \\
\hline $\begin{array}{l}\text { Syriens } \\
\text { et Turcs }\end{array}$ & 96 & 7124 & 45803 & 20400 & 20400 & & & & 93823 \\
\hline Total & 883668 & 852110 & 1006617 & 503981 & 717223 & 80424 & 338726 & 247944 & 4630693 \\
\hline
\end{tabular}

Source : Brasil: 500 anos de povoamento. Rio de Janeiro, IBGE, 2000. Apêndice : Estatísticas de 500 anos de povoamento, p. 226. 
Au début du $\mathrm{XX}^{\mathrm{c}}$ siècle, un courant d'immigration japonaise s'est joint à eux, à l'origine pour fournir des travailleurs aux plantations de café. Une centaine de milliers d'immigrants viennent par ailleurs de ce qui était alors l'Empire ottoman. Pour la plupart libanais et syriens, ils sont encore aujourd'hui appelés de manière générique "Turcos".

\section{Figure 7. Les principaux apports migratoires au XX' siècle}

\section{Figure 8. Les descendants des immigrants au Brésil}


Les nationalités les plus représentées dans la population brésilienne sont donc encore, on l'a vu, celle des pays d'où sont venus les immigrants. On peut y ajouter d'autres groupes nationaux représentant des communautés moins nombreuses et dont l'implantation dans le pays n'est pas exactement la même. Les Autrichiens et les Hongrois suivent à peu de chose près la même répartition que les Allemands. Quant aux Hollandais, ils sont beaucoup plus dispersés dans le pays, en bonne partie parce qu'il s'agit d'une immigration se destinant à l'agriculture et qui a trouvé de bonnes terres dans les États de la périphérie du Centre comme le Minas Gerais ou le Paraná. Les Turcs stricto sensu sont groupés à Rio et São Paulo, mais les Turcos libanais et syriens se dispersent dans tout le Sud, et même dans certains États plus au nord, où ils sont en général commerçants.

\section{Les autres apports migratoires au Brésil}

Lévolution récente a apporté des changements sensibles à cette répartition issue de l'immigration historique. Cette dernière reste d'autant plus dominante que la plupart des étrangers se sont fixés depuis longtemps dans le pays, comme le montre la figure qui les classe en fonction de leur date d'arrivée au Brésil (cf. figure 9, p. 48) : la majeure partie des migrants sont arrivés avant 1990. Mais de nouveaux courants migratoires se sont dessinés au cours des trois dernières décennies. Ils font plus de place aux échanges avec les pays voisins et à de nouveaux courants migratoires.

Les pays voisins, à l'exception de l'Argentine - même si leurs ressortissants n'étaient pas absents du territoire brésilien -, n'ont compté parmi les groupes étrangers les plus nombreux qu'à partir du recensement de 2000. On les trouve, comme on pouvait s'y attendre, dans les

Ces migrations de voisinage
tendent à se développer
grâce à la multiplication
des points de passage,
l’amélioration des
infrastructures routières,
et surtout parce que le
développement économique
du Brésil, plus rapide
que chez ses voisins,
en fait un foyer d’attraction
dans la région.
régions frontalières de chacun des pays, mais aussi et parfois en plus grand nombre dans les grandes capitales du Sud-Est. Les seuls qui se dispersent dans une bonne partie du pays sont les Boliviens et les Paraguayens que l'on retrouve dans tout l'ouest du Brésil.

Ces migrations de voisinage tendent à se développer grâce à la multiplication des points de passage, l'amélioration des infrastructures routières, et surtout parce que 


\section{Figure 9. Les immigrants par date dlarrivée au Brésil}

le développement économique du Brésil, plus rapide que chez ses voisins, en fait un foyer d'attraction dans la région.

Les ressortissants des autres pays étrangers, dont rend compte le recensement de 2000, se répartissent principalement dans les capitales des États fédérés et dans les grandes villes du Sudeste, mais on en trouve aussi de petites concentrations dans des régions plus inattendues : en Amazonie notamment, pour un certain nombre de citoyens des États-Unis, ou dans le Nordeste pour les Belges.

Ces autres nationalités ont des répartitions sur le territoire national assez semblables entre elles et proches de celles de la population brésilienne : leurs ressortissants se distribuent principalement dans le Sud et le Sud-Est, et tout particulièrement dans les grandes capitales, Rio et São Paulo. Le seul pays à faire exception, à double titre, est l'Angola, la seule nation africaine à apparaître avec des effectifs significatifs, et aussi la seule à avoir une certaine dispersion dans les États situés au nord des deux capitales. On peut sans doute l'expliquer par le fait que bon nombre de ces Angolais ont probablement quitté le pays lors de l'indépendance et ont cherché à s'installer dans un pays lusophone où beaucoup de terre était disponible, d'où leur choix du Brésil. 
Figure 10. Les ressortissants des pays voisins du Brésil 


\section{Tendances récentes}

En attendant le recensement de la population de 2010, on ne dispose pas de sources aussi précises sur les tendances récentes de l'immigration au Brésil. On peut toutefois s'en faire une idée en observant les demandes d'autorisation d'immigrer qui ont été enregitrées par les autorités brésiliennes, en l'occurence le ministère du Travail (cf. figure 12). Il ne s'agit évidemment que d'une partie des flux, ceux qui sont traités de façon officielle, ce qui laisse de côté toute l'immigration clandestine ou semi-clandestine.

\section{Figure 12. Autorisations dimmigrer, 2004-2008}

Ces données, que l'on peut transformer en carte de flux, montrent que dorénavant le Brésil attire des migrants du monde entier, et pas seulement des zones qui traditionnellement se dirigeaient vers lui. Aux flux traditionnels venus d'Europe s'ajoutent désormais des courants venus du continent américain, du Nord comme du Sud, et de plus en plus de flux venus d'Asie, principalement du Japon et de Corée. Le seul continent absent est l'Afrique, qui a si longtemps envoyé des migrants involontaires vers le Brésil, du temps de la traite négrière. Les tentatives de rapprochement du Brésil avec l'Afrique, entreprises principalement depuis l'arrivée au pouvoir du président Lula, ne se traduisent pas - ou pas encore - par des flux migratoires. 


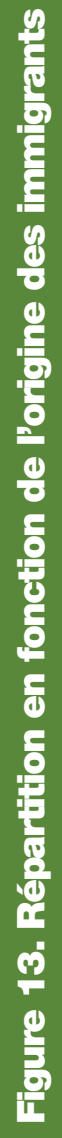


52 Dossier | France - Brésil, sous l'angle des migrations et de l'altérité | 
Les destinations choisies à l'intérieur du Brésil sont diverses selon les origines des migrants : São Paulo, la capitale économique, attire de partout ; le Nordeste semble attirer tout particulièrement les Européens. La nouveauté la plus remarquable est l'apparition de migrants asiatiques : aux Japonais s'ajoutent des Coréens, des Chinois et, plus récemment, des Indiens. Leur répartition dans le pays est semblable à celle des autres nationalités, à l'exception près d'un flux de quelques centaines de Japonais qui se dirigent vers l'Amazonas, ce qui s'explique par la présence de la zone franche de Manaus, où sont installées de nombreuses entreprises japonaises.

Le Brésil redevient donc un pays d'immigration, il attire de plus en plus de migrants qui choisissent de s'y installer parce qu'ils pensent que le développement économique de ce pays est plus rapide que celui de leur propre pays d'origine. Ce ne sont donc plus des migrations organisées vers les plantations et les zones de colonisation agricole, mais des migrations individuelles motivées par une volonté d'ascension sociale. Face à cette situation nouvelle, quelle politique le gouvernement brésilien va-t-il adopter? On en a une idée par une initiative récente : le président Luiz Inácio Lula Da Silva a promulgué le 2 juillet $2009^{(3)}$ la loi

1.664-D,

de 2007, dite "loi de l'amnistie migratoire", qui amnistie les étrangers en situation irrégulière au Brésil. La nouvelle loi permet à tous les étrangers en situation irrégulière, entrés au Brésil avant le $1^{\text {er }}$ février de cette année, de régulariser leur situation. Elle leur accorde la liberté de circulation, le droit de travailler, l'accès à la santé, à l'éducation publique et à la justice. Cette mesure concerne les personnes entrées irrégulièrement au Brésil, dont le visa d'entrée était périmé ou qui n'ont pas bénéficié de la dernière loi d'amnistie migratoire, en 1998, soit, selon les calculs du ministère de la Justice, autour de 50000 personnes (certaines institutions internationales estiment à 200000 le nombre total d'étrangers en situation irrégulière au Brésil). Outre les Boliviens, les Chinois, les Paraguayens, les Péruviens et les Russes sont les principaux groupes concernés.

Le gouvernement brésilien estime qu'aujourd'hui près de quatre millions de Brésiliens vivent à l'étranger, et le secrétaire d'État à la Justice Romeu Tuma Júnior espère que l'initiative brésilienne pourra sensibiliser d'autres pays et "susciter une réciprocité". Selon lui, si beaucoup de pays continuent de criminaliser le traitement des flux migratoires, le Brésil a choisi d'humaniser sa politique d'accueil des étrangers.

\section{Notes}

1. 'Institut national de la statistique et des études économiques, http://www.insee.fr/fr/default.asp.

2. Instituto Brasileiro de Geografia e Estatística, http://www.ibge.gov.br/home/.

3. http://www.agenciabrasil.gov.br/noticias/2009/07/02/materia.2009-0702.0554961635/view, consulté le 18/8/09. 